\title{
The Effect of a Close Binary upon Stellar Pulsation
}

\author{
H. Shibahashi \\ Department of Astronomy, University of Tokyo, Japan
}

\begin{abstract}
Through tidal deformation of the equilibrium state, the pmode oscillations may show apparently complicated features. I discuss the fundamental characteristics of the tidally forced oscillation, and show the possibility that it leads to a triplet fine structure in the frequency spectrum of p-modes.
\end{abstract}

\section{Tidally forced oscillation}

Some pulsating stars are recently realized to be one component of a close binary system (e.g., Paparo et al., 1996). In a close binary system, each component is subjected to a varying external gravitational field due to the other companion. This periodically changing gravitational force induces a tidally forced oscillation, sometimes called the dynamical tide. If the stellar rotation is synchronized with the orbital motion, the tidal force does not induce oscillatory motion but deforms the stellar configuration in hydrostatic equilibrium. This tide is called the equilibrium tide.

An example of the numerical solution of the equilibrium tide for $\ell=2$ is shown in Fig. 1 for the ZAMS model of a $5 M_{\odot}$ star. In the same figure, an example of the tidally forced oscillation of $\ell=2$ is also shown for the same model. Apparently, the amplitude of the dynamical tide shows the oscillatory behavior around the equilibrium tide. For the orbital frequency close to one of the eigenfrequencies, however, the amplitude of the dynamical tide is much larger than that of the equilibrium tide. This is the resonance. Fig. 2 shows the dependence of the amplitude of the quadrupole $(\ell=2)$ dynamical tide upon the frequency. The abscissa is the square of the frequency normalized by $G M / R^{3}$.

It should be remarked that the frequency of the tidally forced oscillation is much lower than the p-mode frequency range and that, with the decrease of $\omega^{2}$, the g-mode eigenspectrum become so dense that resonances become more likely to occur. Hence, there are many chances even for each of the binary components to be elongated by its companion.

\section{Influence of elongation upon the p-mode oscillations}

Let us assume that the $\kappa$ mechanism is working to excite p-mode oscillations in the elongated component. We assume that the star would pulsate radially in the absence of deformation. 

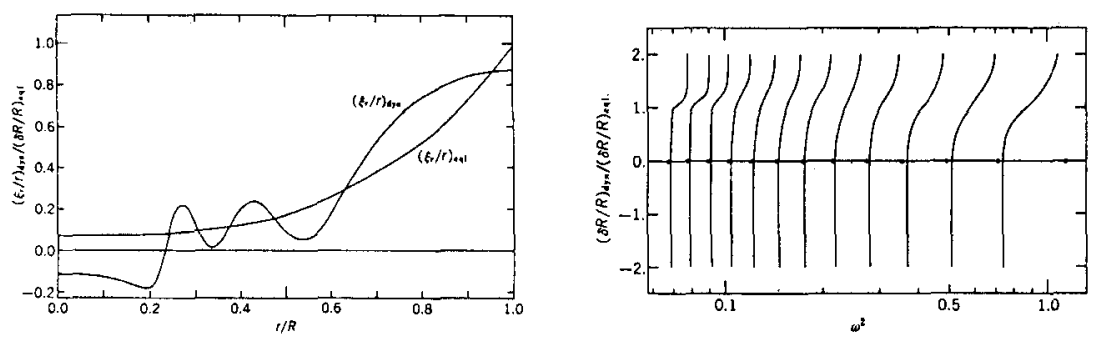

Figure 1. Left: Relative amplitude of the radial displacement of the equilibrium tide $(\ell=2)$ and that of the dynamical tide $(\ell=2)$ with $\omega^{2}=0.40$. Right: The dependence of the amplitude of the dynamical tide $(\ell=2)$ upon the frequency. The ordinate is the relative amplitude at the surface normalized by the corresponding value for the equilibrium tide. After Unno et al. (1979).

While, in the case of dynamical tide, the configuration is changing with time, the timescale is much longer than the p-mode period. Hence, as far as we consider the p-mode oscillations, the tidal deformation can be treated as if it were a static one. Being influenced by elongation of the stellar configuration, the eigenmode is deformed to have an axially symmetric quadrupole component, whose symmetric axis coincides with the direction to the companion star. Hence, the eigenfunction at the surface is characterized by means of a superposition of the spherical harmonic with $\ell=m=0$ and that of $\ell=2$ and $m=0$ with respect to the direction to the companion star.

The aspect angle of the elongation axis varies with the orbital motion of the companion. Therefore, the contribution of the quadrupole component of the eigenfunction to the apparent intensity variation changes with time and produces a triplet fine structure with an equal spacing of the double of orbital frequency in the power spectrum. This is a variation of the "oblique pulsator model", which has explained many observational features of roAp stars.

From the above consideration, I give the following theoretical predictions: a) Even a massive component of a binary can be elongated by its less massive companion. b) In the pulsating star being elongated by its companion, the eigenmode, which would be a pure radial mode in the absence of deformation, is deformed to have a quadrupole component. c) A triplet fine structure with an equal spacing of twice the orbital frequency appears in the frequency spectrum. d) From the amplitude ratio among the triplet peaks, the geometrical configuration can be determined.

\section{References}

Paparo, M., et al. 1996, A\&A, 315, 400

Unno, W., Osaki, Y., Ando, H., \& Shibahashi, H. 1979, Nonradial Oscillations of Stars, (University of Tokyo Press) 